\title{
Photodynamic Therapy for Head and Neck Lesions in the Subtropics
}

\author{
Presented by Francisco Civantos, MD, FACS, University of Miami Miller School of Medicine, Miami, Florida
}

\begin{abstract}
Surgery, radiotherapy, and chemotherapy remain standard treatment options for patients with head and neck carcinomas, but because they are often associated with complications, there is room for improvement. Although not widely practiced and still off-label, photodynamic therapy (PDT) is a promising alternative for superficial malignant or premalignant lesions of the upper aerodigestive tract. It also may be useful for those with recurrent surface disease after surgery or radiotherapy. After an historic look at the data on PDT for head and neck tumors, this article offers a current perspective from a fairly selective PDT program that has achieved dramatic lasting responses. Where PDT may fit in the treatment spectrum for head and neck cancers is one of the unanswered questions requiring further study. (JNCCN 2012;10[Suppl 2]:S65-S68)
\end{abstract}

Dysplastic lesions of the oral cavity and larynx are extremely common and are largely being ignored, declared Francisco Civantos, MD, FACS, Co-Director of the Division of Head and Neck Surgery at the University of Miami Miller School of Medicine, Miami, Florida. Current standard treatment of oral cavity cancer, especially in its early stages, is resection; a combination of therapeutic options, including surgery, radiotherapy, and chemotherapy, is used for later-stage oral cavity cancers. However, these mainstream approaches may be associated with considerable complications. ${ }^{1}$ Therefore, novel

Presented by Francisco Civantos, MD, FACS, Co-Director, Division of Head and Neck Surgery, University of Miami Miller School of Medicine; Chief of Otolaryngology, Sylvester Comprehensive Cancer Center, Miami, Florida.

Dr. Civantos disclosed that he is a consultant for Pinnacle Biologics, Inc.

Correspondence: Francisco Civantos, MD, FACS, UMHC/Sylvester, 1475 NW 12th Avenue, 4th Floor, Miami, FL 33163.

E-mail: fcivanto@med.miami.edu effective alternatives that reduce long-term morbidity without compromising future treatment options, such as photodynamic therapy (PDT), may play an emerging role in managing head and neck tumors.

"We felt the niche for PDT was in recurrent surface disease after previous surgery or radiation, most commonly as adjuvant treatment after initial debulking," stated Dr. Civantos, who has 7 years of experience performing PDT for head and neck lesions at the University of Miami. Unlike radical radiotherapy and chemotherapy, PDT can be performed more than once in cases of recurrent disease or second primaries within the irradiated field. ${ }^{1}$ PDT can also potentially be used for recurrent lesions after surgery, for radiation failure in early laryngeal cancer, and before surgery to limit the amount of resection needed.

\section{Pioneers in PDT for Head and Neck Tumors}

The data on PDT in head and neck go back as far as those in any other site, revealed Dr. Civantos. In the 1990s, several reports appeared on the use of PDT in the treatment of malignancies of the head and neck..$^{2-4}$ One group from France used PDT to treat superficial vocal cord carcinoma in 32 patients, with responses shown in 25 of them. ${ }^{2}$ Another group from the University of Illinois College of Medicine treated 26 patients with biopsy-proven squamous cell carcinoma of the head and neck that failed to respond to traditional treatments or who refused conventional therapies. ${ }^{3}$ Twenty of these patients experienced a complete histologic response (77\%) and 5 (19\%) experienced a partial response for up to 48 months.

Nearly 10 years after these early findings, Biel, ${ }^{4}$ another pioneer in PDT for head and neck malignancies 
and the mentor of Dr. Civantos, presented data from a retrospective review of the medical literature of more than 500 patients treated with PDT, and from his own results in 107 patients. Twenty-five patients with carcinoma in situ and T1 squamous cell carcinoma of the true vocal cord experienced a complete response to a single PDT treatment. Only one recurrence was documented at the time, for a cure rate of $95 \%$ at 79 -month follow-up. According to Dr. Civantos, the light dose varied among these studies, and excellent functional results (quality of voice and oral function) were obtained.

More recent data seem to support these preliminary results with PDT for early-stage tumors of the oral cavity and oropharynx..$^{5-7}$ Results of 2 smaller studies-one a prospective clinical trial ${ }^{5}$ and the other a retrospective review ${ }^{6}$ - support PDT as a potentially curative alternative for early-stage oral tumors. In the prospective trial, the clinical appearance of the oral mucosa in the site of the primary tumor was completely normal in 26 of the 38 patients treated with PDT. ${ }^{5}$ In the retrospective study, 24 of the 30 patients who received PDT experienced complete remission. ${ }^{6}$ In a larger report on 170 patients with 226 lesions, the overall response rate to PDT was approximately $90 \%$, with a complete response rate of more than $70 \%{ }^{?}$

Finally, the use of PDT was explored in patients with end-stage head and neck squamous cell carcinoma for whom other treatment options had been exhausted. ${ }^{8}$ Of the 39 patients treated with PDT, the response rate was $68 \%$. Moreover, the median survival was longer for responders ( 37 months) than for nonresponders (7 months).

\section{The University of Miami Experience}

Dr. Civantos shared the findings from his prospective study of PDT for both early lesions and advanced disease. For patients with superficial T1 through T2 squamous cell carcinoma or dysplasia, PDT was used with curative intent, either as the sole treatment or in combination with limited resection/debulking. For those with advanced disease, PDT was used as an adjuvant treatment. "Although we did try a few advanced cancers with PDT, it was not as rewarding [as with early lesions], and that is not where I think the future of PDT is for head and neck cancer," stated Dr. Civantos. "The classic patient for PDT has field cancerization."
A total of 45 patients with neoplastic diseases of the upper aerodigestive tract participated in the study. Approximately half of the patients were men and half were women. The median age of the patients was 66 years. Half of the participants had premalignant disease and the other half had malignancies with at least one area of documented tumor invasion. All patients had experienced a failed response to previous treatment with either radiotherapy or surgery. Also included were 3 patients with advanced disease.

Categorized by tumor site, $53 \%(\mathrm{n}=24)$ had oral cavity lesions and 40\% ( $\mathrm{n}=18)$ had laryngeal lesions. (The remaining 7\% had other lesions.) Based on pathology, $49 \%(n=22)$ had squamous cell carcinoma, 29\% $(\mathrm{n}=13)$ had severe dysplasia, $20 \%$ $(\mathrm{n}=9)$ had carcinoma in situ, and 2\% $(\mathrm{n}=1)$ had a verrucous carcinoma.

The photosensitizing agent, porfimer sodium (Photofrin), was injected intravenously over $5 \mathrm{~min}$ utes; PDT was performed 48 to 72 hours later. If needed, debulking was performed before PDT, although Dr. Civantos admitted that a great deal of debate exists over this issue. Early in the study, corticosteroids were given intraoperatively and postoperatively. However, because of a concern that these agents might interfere with the PDT effect, the protocol was changed to just posttreatment.

\section{Results, Complications, and Long-Term Follow-Up}

For patients with carcinoma in situ/dysplasia, 16 had a complete response, 3 had a partial response, and 3 had no response. For those with squamous cell carcinoma, 11 had a complete response, 2 had a partial response, and 9 had no response. When the results were evaluated by stage, 13 patients with T1 cancer $(70 \%)$ had a complete response, 4 patients with T2 cancer (50\%) had a complete response, and 8 patients with T3/4 cancer (38\%) had a complete response (Figure 1). Moreover, 78\% of those with cancer of the oral cavity/oropharynx had a complete response to PDT, compared with $72 \%$ of those with cancer of the larynx. Dr. Civantos concluded that these responses to PDT were "definitely above and beyond what would be expected."

Dr. Civantos discussed some of the considerations for performing PDT for a head and neck lesion. "In the head and neck, the complex 3-dimensional 


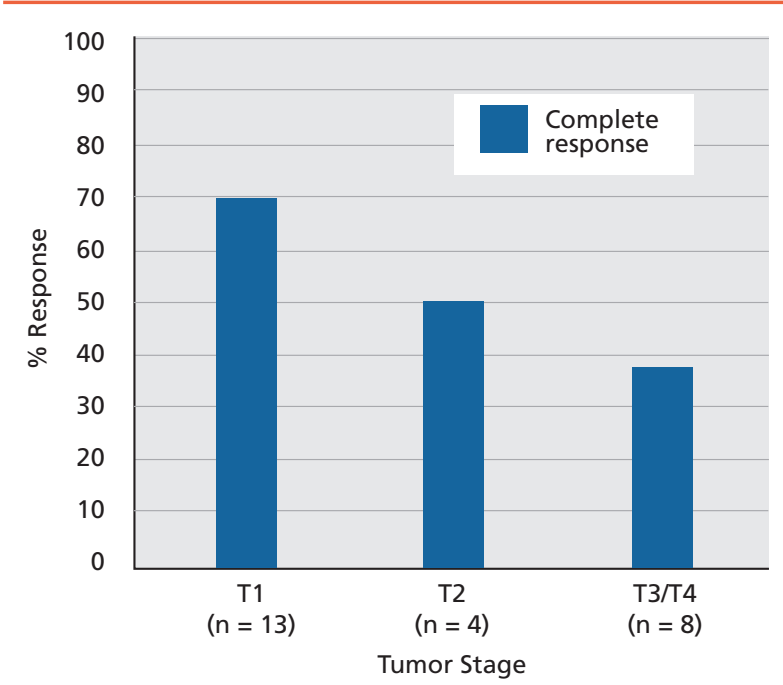

Figure 1 Response to photodynamic therapy (PDT) by tumor stage. More patients with early-stage disease than late-stage disease had a complete response to PDT.

anatomy can make the procedure more tedious and may require significant endoscopic maneuvering," he explained. Through the presentation of a typical type of lesion, raised and exophytic (Figure 2), Dr. Civantos posed a question regarding the timing of debulking: "Is it better to debulk in a previous stage and come back and do PDT, or do it at the same time, which requires it to be dried before treating?" The lesion illustrated in Figure 2 lends itself to treatment with PDT, he added. For this type of lesion, the entire larynx down to the cartilage can be transilluminated with PDT. Another benefit with PDT in this case is the avoidance of radiotherapy. "For in situ and minimally invasive lesions, we tend to hate to shoot the radiation gun, knowing that there is an instance of second primaries," Dr. Civantos reasoned.

The complications linked to PDT during this study are listed in Table 1. Although patients with laryngeal lesions undergoing PDT are relatively painfree afterward, that is not the case with oral cavity lesions. "The difference is striking," stated Dr. Civantos. "Larynx patients go home the same day completely asymptomatic, and oral cavity patients complain of extreme pain for weeks." Some concern was initially expressed about the risk of photosensitivity with PDT, particularly in southern Florida, which has greater sun intensity and duration than in other parts of the country. However, no significant phototoxicity was seen in this study, and, according to Dr. Civantos, "it all boils down to education and proper staffing." Finally, 2 instances were seen of controlled hemorrhage after extensive debulking before PDT, but both were in patients with advanced disease.

The median long-term follow-up of patients in this study was nearly 7 years. A dramatic lasting response was achieved in 30 of 45 patients (71\%), according to Dr. Civantos, and 6 patients required small touch-up endoscopic resections.
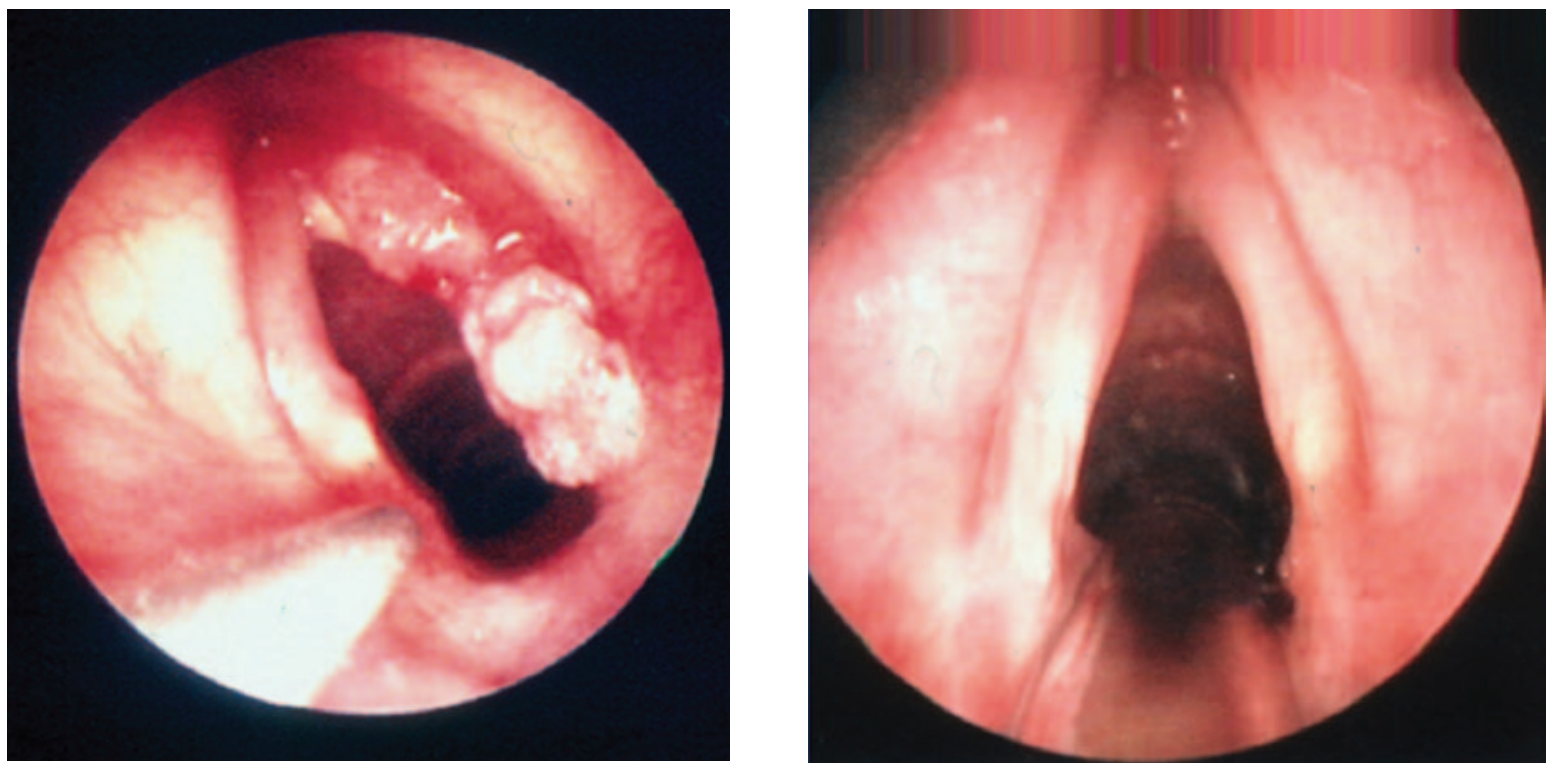

Figure 2 Typical oral cavity cancer treated with photodynamic therapy (PDT). (Left) A raised, exophytic tumor. (Right) The same tumor after PDT. 
Table 1 Complications of Photodynamic Therapy for Oral and Laryngeal Tumors: The University of Miami Experience

\begin{tabular}{|lc|}
\hline Complication & Number $(\mathbf{N}=\mathbf{4 5})$ \\
\hline Significant postoperative pain for oral lesions & 23 \\
Mild skin irritation & 8 \\
Transient second-degree burn on the back of the hand & 1 \\
Inflammatory reaction from extravasation of porfimer sodium at the intravenous site & 1 \\
Transient aspiration & 1 \\
Temporary edema requiring tracheotomy & 1 \\
Unfortunate airway disaster & 1 \\
\hline
\end{tabular}

\section{Moving Forward: Questions Remain}

Dr. Civantos briefly addressed the challenges involved with PDT in head and neck cancers and how he envisions this treatment from a clinical perspective. First is the need for approval of this indication from the FDA. Second is its significant learning curve. "From the technical point of view, we can get at lesions more easily in some ways [in the head and neck], and in other ways, it is more difficult because there are not flat surfaces and straight tubes but a bit more complexity to the anatomy," he added.

In the opinion of Dr. Civantos, perhaps PDT can best be used in patients who have field cancerization and diffuse in situ disease coating the entire lumen of the larynx; these patients might be facing a laryngectomy. However, the superficial, minimally invasive disease could be treated first with PDT. Then, if a small, deeper area of persistent disease is present, it could be removed with a regular cutting or carbon dioxide laser. "Even if there is not a complete response to PDT, there would be a dramatic impact on reducing the extent of surgery," he explained.

Several issues regarding PDT for head and neck tumors require further study, perhaps in a clinical trial. First, why do some patients not experience a response, asked Dr. Civantos. Biologic staging may assist in patient selection for PDT, and reflectance spectroscopy may improve the accuracy of dosimetry. Second, although the voice remains sound with PDT (unlike with laser resection), normative data on voice function and quality are needed to determine whether outcomes in patients with laryngeal lesions are better with PDT than with traditional laser excision, which is Dr. Civantos's prediction.

\section{Conclusions}

Historic data and results from the University of Miami experience suggest a high success rate with PDT for treating superficial malignant or premalignant lesions of the upper aerodigestive tract. A lower success rate with PDT for deeply invasive lesions may be attributed to an inability to illuminate the depth of the tumor. Other potential indications for PDT may involve removing recurrent surface disease before or after surgery. Compared with mainstream treatment options, PDT may reduce long-term morbidity without compromising future treatment options. Clinical trials comparing PDT with standard options such as surgery are needed to confirm the promise of this novel alternative.

\section{References}

1. Nyst HJ, Tan IB, Stewart FA, Balm AJ. Is photodynamic therapy a good alternative to surgery and radiotherapy in the treatment of head and neck cancer? Photodiagnosis Photodyn Ther 2009;6:3-11.

2. Freche $\mathrm{C}, \mathrm{De}$ Corbiere $\mathrm{S}$. Use of photodynamic therapy in the treatment of vocal cord carcinoma. J Photochem Photobiol B 1990;6:291-296.

3. Wenig BL, Kurtzman DM, Grossweiner LI, et al. Photodynamic therapy in the treatment of squamous cell carcinoma of the head and neck. Arch Otolaryngol Head Neck Surg 1990;116:1267-1270.

4. Biel MA. Photodynamic therapy and the treatment of head and neck neoplasia. Laryngoscope 1998;108:1259-1268.

5. Jerjes W, Upile T, Hamdoon Z, et al. Photodynamic therapy outcome for T1/T2 N0 oral squamous cell carcinoma. Lasers Surg Med 2011;43:463-469.

6. Schweitzer VG, Somers ML. PHOTOFRIN-mediated photodynamic therapy for treatment of early stage (Tis-T2NOMO) SqCCa or oral cavity and oropharynx. Lasers Surg Med 2010;42:1-8.

7. Krakullukcu B, van Oudenaarde K, Copper MP, et al. Photodynamic therapy of early stage oral cavity and oropharynx neoplasms: an outcome analysis of 170 patients. Eur Arch Otorhinolaryngol 2011;268:281-288.

8. Tan IB, Dolivet G, Ceruse $P$, et al. Temoporfin-mediated photodynamic therapy in patients with advanced, incurable head and neck cancer: a multicenter study. Head Neck 2010;32:1597-1604. 